\title{
Patients' attitudes towards screening for diabetes and other medical conditions in the dental setting
}

\author{
S. Creanor, ${ }^{* 1}$ B. A. Millward, ${ }^{2}$ A. Demaine, ${ }^{3}$ L. Price, ${ }_{1}^{4}$ W. Smith ${ }_{1}^{5}$ \\ N. Brown ${ }^{5}$ and S. L. Creanor
}



Aim To determine the attitudes of patients attending routine appointments at primary care dental clinics and general dental practices towards the possibility of chair-side screening for medical conditions, including diabetes, in the dental setting. Methods A brief, anonymous, self-administered questionnaire distributed to adult patients ( $\geq 18$ years) attending 2 primary care dental clinics and 16 general dental practices in South-West England. Results One hundred and ninetyseven completed questionnaires were received from patients at primary care dental clinics and 429 from general dental practice patients. Overall, 87\% of respondents thought that it was important or very important that dentists screened patients for medical conditions such as diabetes; $79 \%$ were very willing to let a dental team member carry out screening. The majority indicated willingness to be screened for various medical conditions during a visit to the dentist, with significantly higher proportions of respondents in the primary care clinics indicating willingness (hypertension: 83\% vs 74\%; heart disease: $77 \%$ vs 66\%; diabetes $82 \%$ vs $72 \%$ [all $p<0.02$ ]). Nearly two thirds of primary care clinic respondents and over half of general practice patients indicated that they would be willing to discuss test results with the dental team. Overall, 61\% had never knowingly been screened or tested for diabetes; $20 \%$ reported that they had been tested within the previous 12 months. Conclusion The majority of respondents supported the concept of medical screening in a dental setting and were willing both to have screening tests and discuss their results with the dental team. Patient acceptance is paramount for successful implementation of such screening programmes.

\section{INTRODUCTION}

The prevalence of diabetes is increasing rapidly across the world. In the United Kingdom it was estimated in 2010 that 3.1 million (7.4\%) people aged 16 years and older had diabetes; this is predicted to increase to 4.6 million (9.5\%) by $2030 .{ }^{1}$ Of the 3.1 million, about 850,000 were unrecognised - that is, not included in general practice diabetes

'Centre for Medical Statistics and Bioinformatics, Plymouth University Peninsula Schools of Medicine and Dentistry, Plymouth, UK; ${ }^{2}$ Plymouth Diabetes Centre, Plymouth Hospitals NHS Trust, Derriford Hospital, Plymouth, UK: ${ }^{3}$ Peninsula Medical School, Plymouth University Peninsula Schools of Medicine and Dentistry, Plymouth, UK; ${ }^{4}$ Research Design Service South West, ITTC Building, Tamar Science Park, Plymouth, UK: 5Peninsula Dental Social Enterprise CIC, Plymouth, UK ${ }_{5}^{5}$ Peninsula Dental Social Enterprise CIC, Plymouth, UK; ${ }^{6}$ Peninsula Dental School, Plymouth University Peninsula Schools of Medicine and Dentistry, Plymouth, UK ${ }^{*}$ Correspondence to: Siobhan Creanor

Email: siobhan.creanor@plymouth.ac.uk; Tel: 01752764434

Online article number E2

Refereed Paper - accepted 26 September 2013

DOI: 10.1038/sj.bdj.2013.1247

${ }^{\circ}$ British Dental Journal 2014; 216: E2 registers - but there is no national screening programme in place to help identify these individuals. In addition to those living with undiagnosed diabetes, a further seven million individuals are thought to be at high risk of developing Type 2 diabetes (Type $2 \mathrm{DM}){ }^{2}$ with $\mathrm{HbA}_{1 \mathrm{c}}$ levels above the normal range but not high enough for a diabetes diagnosis, that is, between 42 and $47 \mathrm{mmol} / \mathrm{mol}$ (6.0 and 6.4\%). ${ }^{2-4}$ Evidencebased interventions could prevent or delay the onset of frank diabetes, improving people's health and quality of life, and potentially reduce costs associated with treatment of complications. ${ }^{4-10}$ With the increasing prevalence and associated high economic impact of diabetes, early identification of people at increased risk of Type 2 DM, who could benefit from early intervention, is paramount. ${ }^{11}$

It is usually general medical practitioners (GPs) in the UK who assess diabetes risk in people aged 40-72 years, for example as part of NHS health checks in England. However, these checks are still not offered to many people ${ }^{12}$ and uptake rates remain suboptimal (for example, 45\% in one deprived setting and 24\% of eligible men in an inner-city area). ${ }^{13,14}$ People often only attend their GP when unwell, limiting opportunities to discuss diabetes risk and GPs do not usually screen for diabetes opportunistically. Recent NICE guidelines suggest a number of different venues for Type 2 DM risk-assessment, including dental surgeries, with the guidance that all results of risk-assessments conducted by health professionals outwith general medical practices should be passed on to the person's GP. ${ }^{4}$ Some pharmacists offer opportunistic diabetes screening and a recent study reported on the feasibility of optometrists offering diabetes screening. ${ }^{15}$

Dentists are particularly well-placed to include diabetes risk-assessment as part of their routine care. ${ }^{16}$ In the UK, just over $60 \%$ of dentate adults regularly attend a dentist. ${ }^{17}$ Regular dental visits provide opportunities for prevention and early detection. Recent government policy has encouraged general health promotion 
as part of the role of dental professionals ${ }^{18,19}$ and dental professionals are already involved in preventive health screening and other interventions, such as smoking cessation and oral cancer screening.

There are well-established links between diabetes and oral health, in particular, the bi-directional relationship between diabetes and periodontal disease. ${ }^{20}$ Periodontal disease is three times as prevalent in people with diabetes as people without diabetes ${ }^{21,22}$ and associated with increased cardiovascular morbidity in patients with diabetes. ${ }^{23}$ Patients with Type 2 DM with moderate or severe periodontal disease have increased incidence of end-stage renal disease compared to patients with no or only mild periodontal disease. ${ }^{24}$ Patients with diabetes may have other oral complications including xerostomia, dental caries, candidiasis, burning mouth syndrome, lichen planus and poor wound healing. ${ }^{22,25,26}$

There is little UK research regarding patients' acceptability of dental professionals screening for medical conditions. A recent study within a general medical practice setting, investigating oral health awareness among people with previously diagnosed diabetes, also asked respondents about the idea of dentists being involved in diabetes screening. ${ }^{27}$ Just over half of respondents supported the idea, with almost a quarter reporting they were uncertain. A small number of non-UK studies have reported that the majority of patients support screening for diabetes or other medical conditions in the dental setting ${ }^{28-30}$ and a recent Swedish study reported on the effectiveness of diabetes screening in a dental and primary health care collaboration. ${ }^{31}$ Whether these findings hold true in the UK setting is fundamental, as successful implementation of any offer of screening firstly requires the support of potential users of the service.

As with many novel concepts, initial implementation could take place within dental schools as part of an integrated component of the curriculum, and so the opinions of patients attending primary care dental clinics associated with a UK dental school were sought. However, given that widespread implementation would require the offer of screening in general dental practices, patients attending general dental practices were also surveyed.

The aim of this study, therefore, was to determine the attitudes of patients attending routine appointments at primary care dental clinics and general dental practices towards the possibility of offering chairside screening for diabetes and other medical conditions in the dental setting.

\section{METHODS}

A brief, anonymous, self-administered written questionnaire study was carried out in June 2012 in two primary care dental clinics and between July and August 2012 in general dental practices. The chair of the local NHS Research Ethics Committee confirmed that ethical approval was not required for this study.

\section{Settings and participants}

All adult patients ( $\geq 18$ years) attending routine dental appointments during the study period were eligible to participate. The two primary care dental clinics are run under the auspices of Peninsula Dental School in the South West of England, one being situated in a city area in one of the most deprived wards in England, attracting patients from across the city, the other clinic in a small county city attracting patients both from city and surrounding areas, with significant levels of rural poverty and inequality. A minimum target of 50 completed questionnaires per clinic was set. Local general dental practitioners actively involved with Peninsula Dental School were also invited to participate. The location of most of the dental practices was from the far South West of England, with some practices choosing to participate anonymously. A target of 20 completed questionnaires per practice was set.

\section{Questionnaire}

A one-page, anonymous questionnaire was developed following review of the relevant literature and discussions between the study investigators and following published guidance on survey research. ${ }^{32}$ The draft questionnaire was reviewed by the lay committee of the South-West Peninsula Diabetes Research Network and amended following their suggestions. The revised questionnaire comprised eight items and a free text box to allow for additional comments.

Receptionists distributed the questionnaire to patients attending routine appointments in the dental clinics and practices. Patients were asked to complete the questionnaire before or after their appointments. Completed questionnaires could be placed in a clearly labelled box at the reception or handed back to the receptionist. At the end of the study period, completed questionnaires were returned in person or by mail to one of the study investigators.

\section{Statistical analysis}

Responses were entered into a spreadsheet and exported to Minitab (v16.1, Minitab Inc.) for analysis. Results are presented as percentages, both overall and by patient population (primary care clinic or general practice), based upon the number of responses for the question of interest, given that not all respondents answered all the questions. Statistical analyses were confined to simple cross-tabulations of responses by patient population, with statistical significance of associations assessed using tests of two proportions or chi-squared tests as appropriate; exact p-values were calculated when small expected values were encountered (StatXact v8, Cytel Studio, Cytel Inc). Tests were two-tailed with the significance level set at 5\% and 95\% confidence intervals for between-group differences in proportions were also calculated.

\section{Qualitative analysis}

Patients completing the questionnaire could add spontaneous comments to the pro-forma. These comments were extracted, transcribed and analysed thematically using QRS NVIVO 8 software.

\section{RESULTS}

A total of 197 questionnaires were completed by patients attending the primary care clinics (79 from clinic A, 118 from clinic B). Sixteen general dental practices participated, from which 429 questionnaires were completed, a median of 25 (range 1751) responses per practice. Table 1 summarises the distributions of the responses to each questionnaire item, for all respondents and the two subgroups separately.

\section{Importance of dentists screening for medical conditions}

The vast majority of respondents in both primary care clinics ('clinics') and general practices ('practices') felt it was very important or important for dentists to screen patients for medical conditions (88\% and $87 \%$ respectively). Few respondents indicated that it was not important.

\section{Willingness to have dentist screen for specified medical conditions}

The vast majority of respondents in both clinics and practices indicated that they would be willing to have their dentist perform screening during their visit, with evidence of statistically higher proportions of respondents from clinics compared to respondents from practices, indicating their willingness to be screened for each of high blood pressure (83\% vs 74\%), heart disease (77\% vs 66\%) and diabetes ( $82 \%$ vs $72 \%$ ) (all $\mathrm{p}<0.02$ ).

Willingness to let member of the dental team perform the screening

Again, the vast majority of respondents 
Table 1 Questionnaire results from primary care dental clinics and general dental practices

\begin{tabular}{|c|c|c|c|c|c|c|}
\hline & & $\begin{array}{l}\text { Clinics } \\
(n=197)\end{array}$ & $\begin{array}{l}\text { Practices } \\
(n=429)\end{array}$ & $\begin{array}{l}\text { Overall total } \\
(n=626)\end{array}$ & $\begin{array}{l}95 \% \mathrm{Cl} \text { for difference } \\
\text { (Clinic - Practice) }\end{array}$ & p-value \\
\hline \multirow{4}{*}{$\begin{array}{l}\text { Is it important for a dentist to screen or check } \\
\text { for medical conditions such as diabetes? }\end{array}$} & Very important & $60 \%$ & $53 \%$ & $55 \%$ & $(0.8,15.8) \%$ & \multirow{4}{*}{0.332} \\
\hline & Important & $28 \%$ & $34 \%$ & $32 \%$ & $(13.6,1.9) \%$ & \\
\hline & Unsure & $7 \%$ & $7 \%$ & $7 \%$ & $(4.5,4.2) \%$ & \\
\hline & Not important & $4 \%$ & $6 \%$ & $5 \%$ & $(5.0,2.0) \%$ & \\
\hline \multirow{4}{*}{$\begin{array}{l}\text { Which types of medical conditions would you } \\
\text { be willing to have your dentist screen or check } \\
\text { for during a visit to the dentist? }\end{array}$} & High blood pressure & $83 \%$ & $74 \%$ & $77 \%$ & $(2.2,15.5) \%$ & 0.014 \\
\hline & Heart disease & $77 \%$ & $66 \%$ & $69 \%$ & $(3.3,18.1) \%$ & 0.007 \\
\hline & Diabetes & $82 \%$ & $72 \%$ & $75 \%$ & $(3.3,17.1) \%$ & 0.007 \\
\hline & Other conditions & $37 \%$ & $27 \%$ & $30 \%$ & $(2.1,17.9) \%$ & 0.011 \\
\hline \multirow{3}{*}{$\begin{array}{l}\text { Would you be willing to let a member of the } \\
\text { dental team carry out the screen or check? }\end{array}$} & Very willing & $83 \%$ & $78 \%$ & $79 \%$ & $(1.9,11.3) \%$ & \multirow{3}{*}{0.372} \\
\hline & Not sure & $15 \%$ & $19 \%$ & $18 \%$ & $(9.8,2.8) \%$ & \\
\hline & Not at all willing & $2 \%$ & $3 \%$ & $3 \%$ & $(2.4,3.8) \%$ & \\
\hline \multirow{6}{*}{$\begin{array}{l}\text { Would you prefer any results from the screen } \\
\text { or test to be discussed with you by the dental } \\
\text { team or sent to your GP? }\end{array}$} & Discuss with dental team & $65 \%$ & $53 \%$ & $57 \%$ & $(4.2,20.6) \%$ & \multirow{6}{*}{0.039} \\
\hline & Would depend on test & $11 \%$ & $14 \%$ & $13 \%$ & $(8.4,2.6) \%$ & \\
\hline & GP & $12 \%$ & $21 \%$ & $18 \%$ & $(15.1,3.3) \%$ & \\
\hline & Unsure & $4 \%$ & $4 \%$ & $4 \%$ & $(3.9,2.5) \%$ & \\
\hline & Dental team + GP & $8 \%$ & $7 \%$ & $7 \%$ & $(3.4,5.4) \%$ & \\
\hline & Other combination & $1 \%$ & $2 \%$ & $2 \%$ & $(2.3,2.5) \%$ & \\
\hline \multirow{5}{*}{$\begin{array}{l}\text { Have you ever (knowingly) been screened or } \\
\text { tested for diabetes? }\end{array}$} & No & $67 \%$ & $58 \%$ & $61 \%$ & $(0.2,16.5) \%$ & \multirow{5}{*}{0.003} \\
\hline & In last 6 months & $13 \%$ & $9 \%$ & $10 \%$ & $(1.1,9.9) \%$ & \\
\hline & 6-12 months ago & $6 \%$ & $11 \%$ & $10 \%$ & $(9.5,0.4) \%$ & \\
\hline & More than 12 months & $14 \%$ & $18 \%$ & $17 \%$ & $(10.6,1.6) \%$ & \\
\hline & Yes - unknown when & $0 \%$ & $3 \%$ & $2 \%$ & $(1.4,5.7) \%$ & \\
\hline
\end{tabular}

indicated that they would be very willing to let a member of the dental team carry out the necessary screening or check, with a slightly greater proportion from the clinics indicating their willingness than from practices (83\% vs 78\% respectively). Few respondents were not at all willing.

\section{Discussion of screening results}

Overall, the majority of respondents indicated that they would prefer to discuss the results initially with the dental team (57\%). A significantly higher proportion of respondents from clinics indicated that they would be willing to discuss screening results with the dental team compared with respondents from practices (65\% vs 53\%). A significantly higher proportion of respondents from practices indicated that they would want their results sent to their GP for discussion compared to respondents from clinics (21\% vs 12\%). Similar proportions in clinics and practices indicated they would like to discuss the results with both the dental team and GP (8\% and $7 \%$ respectively) while $4 \%$ of respondents in both clinics and practices indicated that they were unsure.

\section{Previously been screened or tested for diabetes}

Overall the majority reported never knowingly having been tested or screened for diabetes (61\%); however, there was evidence of a difference in the distributions of the responses from clinics compared to practices ( $p=0.003)$. A significantly greater proportion of respondents in clinics reported having never been screened compared to respondents in practices $(67 \%$ vs $58 \%$ ), while a significantly higher proportion of respondents in practices had been tested between 6 and 12 months previously compared to respondents in clinics (11\% vs $6 \%$ ). Overall, around 10\% of respondents had been screened for diabetes in the previous six months.

\section{Additional comments}

Of the 626 participants, 68 provided additional comments. Six stated that they already had a diagnosis of Type 2 DM and a further individual had been informed by the GP of a diagnosis of 'borderline' diabetes. Four reported having been screened or checked for diabetes elsewhere. of the remainder, 12 indicated that they were unequivocally positive about being screened for diabetes in the dental setting and a further 19 were mainly positive but with some reservations or qualifications. In order of frequency, the qualifications mentioned were: as long as there was no cost attached or was part of the NHS provision; as long as well-trained and qualified staff were doing the test - doctors, nurses or dentists were stipulated and the expression 'dental team' was off-putting to some; as long as their GP was informed; as long as confidentiality was protected; as long as they had a good relationship with the dentist; if they had not been checked elsewhere; if oral cancer was included in the screening; if the test was not invasive and as long as performing checks did not place an additional burden on dentists to distract them from providing dental care.

'As life is so busy, it is very helpful to have these screenings done and if it could be done in a dental check it would kill two birds with one stone!' (GP1425)

'If this was being done at the surgery I would only want a qualified nurse or doctor to carry out these tests. Training! Training! And more training!' (GP310) 
'Yes to all as long as it does not cost.' (GP714)

Twenty people did not agree with diabetes checks being carried out in dental surgeries and a further two people were unsure.

'I'm not sure as it seems strange for a dentist to do these checks except for teeth.' (GP612)

of those who disagreed, the most common reason given was that the GP should be the one to carry out screening. Other reasons included a general distaste for screening, expressed as 'If it ain't broke, don't fix it', a conviction that dentists should concentrate on dentistry, concern about cost-effectiveness and use of resources and suspicion about underlying political motives for introducing medical screening in dental settings.

'Is this another government initiative to introduce private health care through the back door? Cynical? Yes probably.' (GP1606)

'A dentist should concentrate on dental issues otherwise there could be a chance the service the patient receives is watered down!' (GP1605)

'Where you have a relationship established with your GP I feel it is an intrusion for others to be screening for the same things. The cost effectiveness is doubtful. Perhaps a record of who is already screened elsewhere would alleviate this.' (GP1112)

Three people expressed confusion about the possible implications for other conditions they had and medications they were on. One person pointed out that many cannot afford dental treatment and would thus be excluded from this service.

\section{DISCUSSION}

This is the first UK study to investigate dental patients' attitudes of and acceptance towards screening for medical conditions, including diabetes, within dental settings. Adult patients attending routine appointments at either primary care dental clinics, run by a community-based dental school, or general dental practices across SouthWest England were surveyed.

The overwhelming majority of respondents agreed it was (very) important for dentists to screen for medical conditions such as diabetes and the majority of respondents were very willing to be screened by a member of the dental team. The majority of respondents were willing to be screened for hypertension, heart disease and diabetes, with three quarters of respondents overall supporting screening for diabetes. The majority of respondents were happy to discuss screening results with the dental team, with just under a fifth preferring to discuss results with their general medical practitioner.

While overall there were favourable responses to most questions from both populations of patients, there were some differences: approximately 12\% more primary care clinic respondents than general practice respondents indicated that they would be happy to discuss the results with the dental team. Similarly, around 10\% more respondents from primary care clinics indicated their willingness to be screened for each of hypertension, heart disease and diabetes compared to respondents from general practices.

The responses to the questionnaire were very similar to those from a US study of dental patients attending either a dental school clinic or one of two private dental practices. ${ }^{29}$ In summary, this US study reported that $94 \%$ of dental school patients and $77 \%$ of private patients thought it was important for dentists to screen patients for medical conditions. Similar high proportions indicated willingness for medical screening or monitoring that yields immediate results (90\% and 76\% respectively), while 90\% of dental school patients and $79 \%$ of private patients reported that they would be happy to discuss the results immediately, during the dental visit. The proportions indicating willingness for the dentist to screen for hypertension, diabetes and heart disease among dental school and private patients were 90\% vs 67\%, 83\% vs 57\% and $82 \%$ vs 57\%, respectively. ${ }^{29}$

There are few other published studies of dental patients' attitudes towards screening for medical conditions. A recent practicebased study assessed the feasibility of blood glucose testing for diagnosis and monitoring of diabetes in 28 dental practices: 24 in the US and 4 in Sweden. ${ }^{28}$ A brief questionnaire was completed by patients at the end of their appointment: 83\% of respondents reported that blood glucose testing was a good idea and 79\% reported that blood glucose testing gave useful information. ${ }^{28} \mathrm{~A}$ feasibility study of diabetes screening at periodontal clinics at a US University College of Dentistry included a brief survey on patients' experiences of the screening process, together with a small number of individual interviews. ${ }^{30}$ Ninety percent of respondents agreed that dental visits were a good place to have glucose testing, supported further by the saving of time by not needing to wait for, and attend, an additional medical appointment. The issue of patient choice about accepting an invitation for screening was noted. A recent UK study investigating the oral health awareness of adult patients attending diabetic clinics included three questions on the potential of dentists' involvement in diabetes care. ${ }^{27}$ Just over half of respondents supported the idea of dentists being involved with diabetes screening, with just under a quarter indicating they were unsure.
However, nearly two thirds of respondents indicated their unwillingness to pay for screening if offered by dentists. ${ }^{27}$

The findings of the study reported here are limited by the anonymous sampling of patients attending routine appointments. As the questionnaire was distributed to willing patients by receptionists in busy dental clinics and practices, information was not collated on refusal rates. In an attempt to keep the response rate as high as possible, the questionnaire was kept very short and therefore demographical information was not requested. This means, therefore, it is not possible to investigate associations between responses and, for example, age group or gender. There may be limited generalisability given the study was only conducted in one geographical area, which may not reflect the UK population overall. However, patients attending the primary care clinics are from diverse social backgrounds and geographical areas, and the participating general dental practices are a mixture of urban, sub-urban and rural practices, with both private and NHS patients. There are inherent limitations of such selfreport studies, in particular the potential for response bias such that patients agreeing to complete the questionnaire are more likely to have strong opinions in one direction or the other. However, the responses generally covered the entire response ranges, for patients from both settings, and the high numbers of participants suggest interest in this topic.

The results of this brief survey indicated that patients attending routine dental appointments support and are willing to participate in medical screening in the dental setting. If this study were to be followed up by any form of sampling for the purposes of screening, there would clearly be the need for additional staff training. Pilot studies would be necessary to address the potential practical difficulties of undertaking such screening. There would also have to be careful consideration regarding the implications of false negative and false positive results, the cost and professional time availability. A small number of US studies have investigated the dental teams' attitudes towards screening for medical conditions and in particular diabetes..$^{30,33,34}$ One study reported that most dentists thought that chair-side screening for medical conditions was important and that they were willing to undertake screening in a dental setting, ${ }^{33}$ while acknowledging that further education was necessary, in particular on how to implement such screening given the perceived barriers of cost and time. In a study of dentists' attitudes relating to diabetes, the majority reported that addressing 
diabetes was important to their role as a dentist; however, fewer than half of the respondents felt that they knew enough about how to assess patients for diabetes. ${ }^{34}$ A recent study reported on dental providers' experiences of screening for diabetes in relation to periodontal visits. ${ }^{30}$ In that study, a number of providers raised the issue of cost, while there were also potential barriers in terms of communication of test results. Overall, however, the dental providers as well as patients believed that the dental visit is an ideal opportunity for diabetes screening. ${ }^{30}$ Further research is certainly needed, therefore, to investigate these challenges within the UK context, with its different healthcare system, together with an assessment of the effectiveness and costeffectiveness of providing such screening in the dental setting, before widespread implementation could be recommended.

\section{CONCLUSION}

Screening for medical conditions, such as diabetes, in dental settings is an approach that is receiving increasing interest. The vast majority of respondents supported the principle of screening for medical conditions in dental settings, were willing to take part in screening and discuss results with the dental team. Patient acceptance is the first critical element for successful implementation of such a strategy.

We would like to thank the reception staff in the primary care dental clinics, the general dental practitioners and their practice receptionists who supported the study, all the patients who completed the questionnaire and the lay committee of the South-West Diabetes Research Network for their ongoing support.

1. Holman N, Forouhi N G, Goyder E, Wild S H. The Association of Public Health Observatories (APHO) Diabetes Prevalence Model: estimates of total diabetes prevalence for England, 2010-2030. Diabet Med 2011; 28: 575-582
2. Diabetes UK. Position statement: impaired glucose regulation (IGR)/non-diabetic hyperglycaemia (NDH)/prediabetes. London: Diabetes UK, 2009.

3. John W G, Hillson R, Alberti S G. Use of haemoglobin $\mathrm{A} 1 \mathrm{c}(\mathrm{HbA1c})$ in the diagnosis of diabetes mellitus. The implementation of World Health Organization (WHO) guidance 2011. Practical Diabetes 2012 : 29: 12-12a.

4. National Institute for Health and Care Excellence. Preventing type 2 diabetes: risk identification and interventions for individuals at high risk. London: NICE, 2012. Online article available at http:// guidance.nice.org.uk/ph38 (accessed October 2013)

5. Tuomilehto J, Lindstrom J, Eriksson J G et al. Prevention of type 2 diabetes mellitus by changes in lifestyle among subjects with impaired glucose tolerance. N Eng/ J Med 2001; 344: 1343-1350.

6. Knowler W C, Barrett-Connor E, Fowler S E et al. Reduction in the incidence of type 2 diabetes with lifestyle intervention or metformin. N Engl J Med 2002; 346: 393-403.

7. Lindström J, Peltonen M, Eriksson J G et al. Determinants for the effectiveness of lifestyle intervention in the Finnish diabetes prevention study. Diabetes Care 2008; 31: 857-862.

8. Li G, Zhang P, Wang J et al. The long-term effect of lifestyle interventions to prevent diabetes in the China Da Qing Diabetes Prevention Study: a 20-year follow-up study. Lancet 2008; 371: 1783-1789.

9. Perreault L Pan 0 , Mather $\mathrm{K} J$ et al. Effect of regression from prediabetes to normal glucose regulation on long-term reduction in diabetes risk: results from the Diabetes Prevention Program Outcomes Study. Lancet 2012: 379: 2243-2251.

10. Zhuo $X$, Zhang $P$, Gregg $E$ W et al. A nationwide community-based lifestyle programme could delay or prevent type 2 diabetes cases and save $\$ 5.7$ billion in 25 years. Health Aff (Millwood) 2012; 31: 50-60.

11. Khunti K, Davies M J. Diabetes prevention: NICE opportunity for implementing programmes in the real-world setting. Diabet Med 2013; 30: 1-2.

12. Diabetes UK. The NHS Health Check Programme: let's get it right. London: Diabetes UK, 2012.

13. Dalton A R, Bottle A, Okoro C, Majeed A, Millett C. Uptake of the NHS Health Checks programme in a deprived, culturally diverse setting: cross-sectional study. J Public Health 2011; 33: 422-429.

14. Lambert A M, Burden A C, Chambers J, Marshall T, Heart of Birmingham Teaching Primary Care Trust. Cardiovascular screening for men at high risk in Heart of Birmingham Teaching Primary Care Trust: the 'Deadly Trio' programme. J Public Health 2012; 34: 73-82.

15. Howse J H, Jones S, Hungin A P. Screening and identifying diabetes in optometric practice: a prospective study. Br J Gen Pract 2011; 61: 436-442.

16. Levine R S. Obesity, diabetes and periodontitis a triangular relationship? Br Dent J 2013; 215: 35-39.

17. Steele J G, O'Sullivan I. Executive summary: Adult
Dental Health Survey 2009. London: Health and Social Care Information Centre, 2010.

18. Department of Health. Modernising NHS dentistry: implementing the plan. London: The Stationery Office, 2000.

19. Department of Health. NHS dentistry: options for change. London: The Stationery Office, 2002.

20. Taylor G W. Bidirectional interrelationships between diabetes and periodontal diseases: an epidemiologic perspective. Ann Periodontol 2001; 6: 99-112.

21. Mealey B L, Oates T W. Diabetes mellitus and periodontal diseases. J Periodonto/ 2006 77: 1289-1303.

22. Preshaw P M. Periodontal disease and diabetes. J Dent 2009; 37: S575-S577.

23. Southerland J H, Moss K, Taylor G W et al. Periodontitis and diabetes associations with measures of atherosclerosis and CHD. Atherosclerosis 2012; 222: 196-201.

24. Shultis W A, Weil E J, Looker H C et al. Effect of periodontitis on overt nephropathy and end-stage renal disease in type 2 diabetes. Diabetes Care 2007; 30: 306-311.

25. Ryan M E. Diagnostic \& therapeutic strategies for the management of the diabetic patient. Compend Contin Educ Dent 2008; 29: 32-44.

26. Hodge P J, Robertson D, Paterson K, Smith G L, Creanor S, Sherriff A. Periodontitis in non-smoking type 1 diabetic adults: a cross-sectional study. J Clin Periodontol 2012; 39: 20-29.

27. Bowyer $V$, Sutcliffe $P$, Ireland $R$ et al. Oral health awareness in adult patients with diabetes: a questionnaire study. Br Dent J 2011; 211: E12

28. Barasch A, Safford M M, Qvist V et al. Random blood glucose testing in dental practice: a communitybased feasibility study from The Dental PracticeBased Research Network. J Am Dent Assoc 2012; 43: 262-269.

29. Greenberg B L, Kantor M L, Jiang S S, Glick M M. Patients' attitudes toward screening for medical conditions in a dental setting. J Public Health Dent 2012; 72: 28-35.

30. Rosedale M, Strauss S. Diabetes screening at the periodontal visit: patient and provider experiences with two screening approaches. Int J Dent Hyg 2012; 10: 250-258.

31. Engstrom S, Berne C, Gahnberg L, Svärdsudd K. Effectiveness of screening for diabetes mellitus in dental health care. Diabet Med 2013; 30: 239-245.

32. Kelley K, Clark B, Brown V, Sitzia J. Good practice in the conduct and reporting of survey research. Int J Qual Health Care 2003; 15: 261-266.

33. Greenberg B L, Glick M, Frantsve-Hawley J, Kantor M L. Dentists' attitudes toward chairside screening for medical conditions. J Am DentAssoc 2010; 141: 52-62.

34. Esmeili T, Ellison J, Walsh M M. Dentists' attitudes and practices related to diabetes in the dental setting. J Public Health Dent 2010; 70: 108-114. 